\title{
Emergent Transcatheter Aortic Valve Implantation as a Life-saving Procedure: A Primary Treatment Approach
}

\author{
Emre Ozdemir, Fatma Kayaalti Esin, Selcen Yakar Tuluce and Mustafa Karaca \\ Department of Cardiology, Katip Celebi University, Ataturk Education and Research Hospital, Cardiology Clinic, Izmir, Turkey
}

\begin{abstract}
Severe aortic stenosis (SAS) is the most common valvular heart disease in elderly patients. Untreated SAS is known to have $50 \%$ mortality within 2 years of onset of symptoms. SAS with acute decompensated heart failure is fatal and requires immediate, appropriate treatment. Urgent transcatheter aortic valve implantation (TAVI) is acceptable with feasible outcomes for a selected group of patients with decompensated SAS. Herein, we present a case of a primary TAVI procedure with a good outcome for decompensated SAS. The patient was an 85-year female with SAS with peak/mean transvalvular gradients of $73 / 42 \mathrm{mmHg}$, and left ventricular ejection fraction (LVEF) of $60 \%$ on echocardiography. The TAVI procedure was confirmed with echocardiography and angiography. As there was no anatomic contraindication for TAVI, primary TAVI was selected for this patient with implantation of a No. 29 Portico ${ }^{\mathrm{TM}}$ TAVI valve (Abbott; St. Jude Medical Inc., St. Paul, MN, USA). To the best of the authors' knowledge, this case is the first to be reported as primary TAVI which was performed in 4 hours of emergency situation. A life-saving procedure was performed, which lasted 4 hours from door-tovalve placement.
\end{abstract}

Key Words: Acute decompensated heart failure, Aortic valve stenosis, Cardiogenic shock, Transcatheter aortic valve implantation.

How to cite this article: Ozdemir E, Esin FK, Tuluce SY, Karaca M. Emergent transcatheter aortic valve implantation as a life-saving procedure: a primary treatment approach. J Coll Physicians Surg Pak 2019; 29(10):996-9.

\section{INTRODUCTION}

Severe aortic stenosis (SAS) is the most common valvular heart disease in elderly patients. ${ }^{1}$ Untreated SAS is known to have $50 \%$ mortality within 2 years of the onset of symptoms. SAS with acute decompensated heart failure increases this mortality rate, but appropriate treatment can be applied. ${ }^{2}$ The main treatment option for SAS is surgical aortic valve replacement (SAVR), but because of advanced age and high surgical risk, $40.5 \%$ patients with SAS could not be operated until the introduction of transcatheter aortic valve implantation (TAVI). ${ }^{3}$ TAVI treatment was first successfully performed on humans with SAS by Cribier et al. in 2002 and since then, has become a cornerstone for patients at high surgical risk or who were inoperable, and is now widely applied worldwide with ongoing developments. ${ }^{4}$ Ideally, TAVI should be performed as an elective procedure for patients with SAS after they have been carefully monitored. However, emergent TAVI is feasible with acceptable clinical outcomes for a selected group of patients with decompensated SAS.

We, herein present a case of an emergent TAVI procedure with a good clinical outcome in an 85-year female patient with SAS and severe coronary artery disease who presented

Correspondence: Dr. Emre Ozdemir, Katip Celebi University, Ataturk Education and Research Hospital, Izmir, Turkey

E-mail: emreozdemir27@yahoo.com.tr

Received: October 29, 2018; Revised: March 11, 2019;

Accepted: March 28, 2019 at the Emergency Department (ED) with decompensation and poor hemodynamic parameters.

\section{CASE REPORT}

A 85-year female with SAS presented at the ED with dyspnea and poor clinical status. In the ED, hypotension and hypoxia were determined. The patient was intubated after successfully performing cardiopulmonary resuscitation following a cardiopulmonary arrest with dopamine infusion being started as supportive treatment. Crepitant rales were determined in the basal and mid zones on auscultation due to lung edema. Blood pressure was $80 / 50 \mathrm{mmHg}$ despite the dopamine infusion. Troponin I levels were negative at 0.014 and $0.026 \mu \mathrm{g} / \mathrm{L}$, with a cutoff value of $0-00.6$. There were $90 \%$ stenosis of left anterior descending (LAD) artery, $90 \%$ of left circumflex (LCX) and a chronically occluded right coronary artery (RCA), with tortuous coronary anatomy and mid-distal lesions, on a recent coronary angiography from another medical centre.

Echocardiographic evaluation showed SAS with peak/ mean transvalvular gradients of $73 / 42 \mathrm{mmHg}$, and left ventricular ejection fraction (LVEF) of $60 \%$. After this angiography and echocardiography in the other centre, the patient had been considered inoperable because of the high surgical risk. Electrocardiogram (ECG) showed sinus rhythm and left ventricular hypertrophy with signs of strain and rare ventricular extra systole. Due to the ongoing unstable hemodynamic parameters in the presence of normal LVEF and ineffective inotropic 
support, an emergent consultation between the cardiology and cardiovascular surgery clinics agreed by consensus that because of the high SAVR risk, a TAVI procedure was acceptable to rescue the patient from cardiogenic shock. There was no time to perform a computed tomography scan for evaluation. With the benefit of the patient intubated, cardiac catherisation was performed under general anesthesia using transesophagial echocardiography (TEE). A 7-F sheath was placed through the left femoral artery. Aortic diameters were measured using TEE and aortography. The aortic valve perimeter and aortic annulus diameter were calculated as $77 \mathrm{~mm}$ and $27 \mathrm{~mm}$, respectively on 2-D and 3-D TEE and the aortic annulus diameter and the ascending aortic diameters were calculated as 25.9 and $29.8 \mathrm{~mm}$ via angiography (Figure 1). After this step, the suitability of the peripheral vessels for the TAVI procedure was confirmed with peripheral angiography (Figure 2); and since there was no anatomic contraindication for TAVI, emergent TAVI was performed with a No. $29 \mathrm{~mm}$ Portico ${ }^{\mathrm{TM}}$ TAVI valve (Abbott; St. Jude Medical Inc.,St. Paul, MN, USA) (Figures 3a-c). A transient cardiac pacemaker lead was positioned in the right ventricular apex from the 6-F sheath in the left femoral vein. Two Perclose ProGlide ${ }^{\circledR}$ closure devices (Abbott Vascular Medical, St. Paul, MN, USA) were placed under the scopic punching to the right main femoral artery. After observation of a post-procedure moderate paravalvular aortic insufficiency, the periprocedural TEE showed that the non-coronary side of the valve was not sufficiently open (Figure $3 d$ ) causing moderate paravalvular leakage. Post-dilatation with a $25 \mathrm{~mm}$ balloon

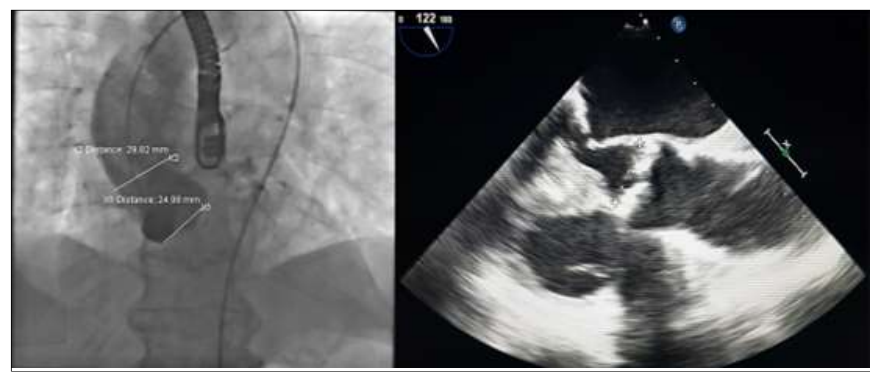

Figure 1: On the left side, the angiographic aortic calculation for transcatheter aortic valve implant (TAVI), and on the right side the calculation on transesophagial echocardiography (TEE) with similar sizes suitable for No. 29 TAVI valve.

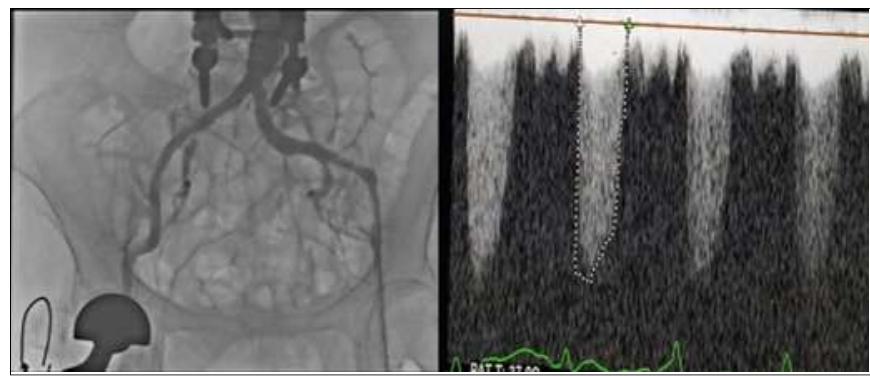

Figure 2: On the left side, peripheral angiography shows atherosclerotic changes but suitable for TAVI, and on the right side the gradient on the aortic valve is $73 / 42 \mathrm{mmHg}$.

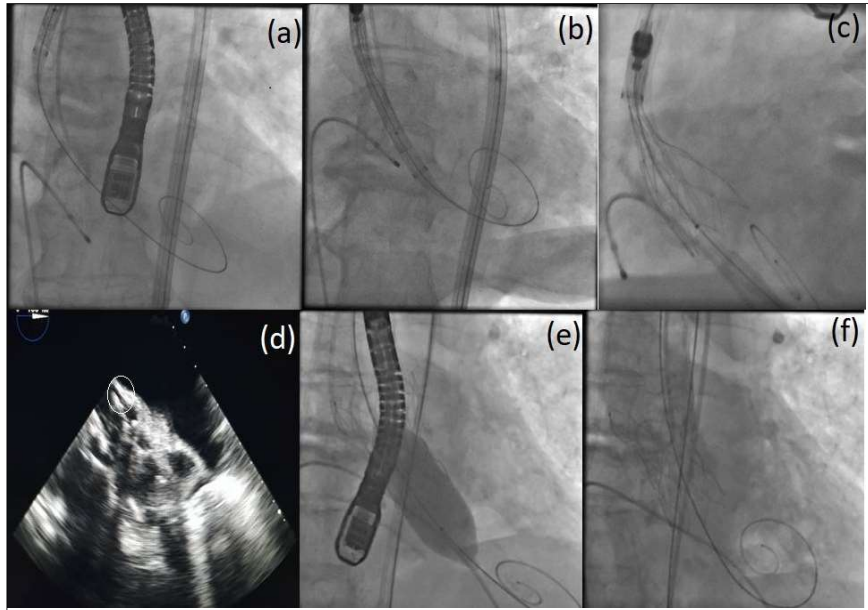

Figure 3: Transcatheter aortic valve implantation (TAVI) delivery system carried forward over the SafariTM guidewire to the aortic annulus (a-b). After the positioning of the delivery system with aortography, a No. 29 Portico $^{\mathrm{TM}}$ (St. Jude Medical Inc., St. Paul, MN, USA) prosthetic TAVI valve was implanted successfully to the stenotic valve area step-by-step (c). After observation of malaposition of the non-coronary side of the TAVI valve (in the white circle) on TEE (d). post-dilatation with a $25 \mathrm{~mm}$ balloon was performed (e). Paravalvular leakage disappeared after post-dilatation (f)

(Figure 3e) was performed, with blood pressure decreasing by rapid RV pacing. The paravalvular leakage was resolved after post-dilatation (Figure $3 f$ ). Post-implantation peak/mean transvalvular gradients of $22 / 12 \mathrm{mmHg}$ and a full-functional TAVI valve were seen on 2-D TEE. The puncture sites were successfully closed with the previously placed Perclose ProGlide ${ }^{\circledR}$. No extravasation was observed on the control peripheral angiography. The patient was given $300 \mathrm{mg}$ acetylsalicylic acid; and $450 \mathrm{mg}$ clopidogrel under the operator initiative, with the idea that thromo-boembolic events could not be tolerated due to the patient's clinic status. The patient's hemodynamics rapidly improved and the dopamine infusion requirement was reduced. Postprocedure, the patient was extubated in two hours and was totally in a good condition at the 7 th hour. As hemoglobin decreased from 11 to $8.8 \mathrm{~g} / \mathrm{dL}, 2$ units of erythrocytes were transfused. There was a minimal increase of the serum creatinine level from 0.93 to 1.25 $\mathrm{mg} / \mathrm{dL}$ but there was no need for hemodialysis. Therefore, the emergent TAVI procedure, which was performed successfully within four hours of door-tovalve time, was a life-saving procedure.

\section{DISCUSSION}

Acute decompensated symptomatic SAS must be treated immediately, but it is a gruelling process. The gold standard procedure of SAVR has high mortality rates for acute decompensated SAS in advanced-age patients with 30 -day mortality reported up to $10.1 \% .^{2}$ TAVI is a treatment option for high-risk patients with SAS, but there are no definitive reports for acute decompensated SAS. ${ }^{5}$ Also with restricted datas, 30-day mortality in patients with decompensated SAS is reported to be $10.1 \%$ in surgery and $4.8 \%$ in TAVI. 6 
Kashiyama N. et al. presented a case report of emergent TAVI with a $23 \mathrm{~mm}$ Edwards-SAPIEN valve. 6 The shortterm outcome after emergent TAVI suggests that it is a reasonable treatment option for acute decompensated SAS patients who are resistant to medical therapy and at high risk for SAVR.7

In the 2014 American Heart Association/American College of Cardiology (AHA/ACC) guidelines, there is a class IIb evidence and $C$ level recommendation that the balloon valvuloplasty (BAV) procedure can be used as a bridge to SAVR or TAVI in patients with symptomatic SAS.8 However, SAS patients with acute decompensation not treated medically, may also undergo BAV, which is known to have limited success secondary to re-stenosis and clinical deterioration in most patients within 3 to 6 months or with complications such as aortic insufficiency. Mortality after BAV has been reported to be up to $17 \% .9$

In the TVT registry, it is recorded that emergent TAVI procedure was performed in 3,953 (9.9\% of all registry) patients between November 2011 and June 2016. Lifethreatening bleeding, major vascular complications, Myocardial infarction (MI), stroke, new permanent pacemaker, a requirement for SAVR, and paravalvular regurgitation rates were similar in all groups; whereas, acute kidney injury (AKI) was observed more frequently in the emergent TAVI group than in the elective group (8.2\% vs. $4.2 \%$; $\mathrm{p}<0.001$ ); but this difference was not determined to be significant after baseline characteristics adjustment. Higher mortality rates at 30 days and 1 year were determined in the emergent TAVI group and the in-hospital mortality rate was $6.1 \% .10$

In the current case, there was hyperdynamic left ventricle (LV) due to dopamine infusion with low systemic blood pressure under cardiogenic shock and the patient was decompensated because of LV outflow tract obstruction with SAS. The patient did not respond to medical treatment.

The accepted standard strategy of BAV plus percutaneous coronary intervention (PCI) of LCX and LAD has been proven to be a good option for this patient when the two control troponin I values were negative, and so in the current case it was decided that this was not a coronary event. However, regarding the other option of BAV plus TAVI, as there was the possibility of unwanted acute aortic insufficiency, it was decided on the basis of previous experience that an emergent TAVI procedure should be applied and it was performed successfully instead of BAV, with a 4-hour door-to-valve time, as a life-saving procedure.

There was no further need for inotropic medicines or supportive treatment for hemodynamics after the TAVI valve was implanted. Post-procedure, the patient was extubated in two hours and was totally in good condition at the $7^{\text {th }}$ hour.
After this TAVI procedure, for the critical coronary artery disease, because of tortuosity, mid-distal lesions and the good general health of the patient, it was decided that medical treatment for coronary disease should be applied first.

In experienced centres, TAVI may be a better and safer option than BAV when there is no response to medical treatment for aortic stenosis with hemodynamic impairment and poor general condition. In the current case, it was considered that the SAS had to be treated quickly and successfully with an emergent TAVI procedure to affect the prognosis positively. The increasing age of patients and the increased need for TAVI will lead to valve interventions in the future in emergency situations to become a reality and a new term of "Primary TAVI" will come into use.

In conclusion, although BAV procedure is recommended as a bridge to SAVR or TAVI in patients with symptomatic SAS, emergent TAVI procedure can be a lifesaving procedure in experienced centres, without any loss of time. There is a need for further studies to define the role of primary TAVI in such situations. Similar to the door-to-balloon time for primary PCls, there could be a future definition for TAVI of door-to-valve time.

\section{PATIENTS' CONSENT:}

All procedures were performed with the consent of the patient.

\section{CONFLICT OF INTEREST:}

Authors declared no conflict of interest.

\section{AUTHORS' CONTRIBUTION:}

EÖ: Data collection, writing.

FKE, SYT: Helped supervise.

MK: Reviewed, supervised.

\section{REFERENCES}

1. Nkomo VT, Gardin JM, Skelton TN, Gottdiener JS, Scott CG, Enriquez-Sarano M. Burden of valvular heart diseases: A population-based study. Lancet 2006; 368:1005-11.

2. McHenry MM, Rice J, Matlof HJ, Flamm MD Jr. Pulmonary hypertension and sudden death in aortic stenosis. Br Heart $J$ 1979; 41:463-7.

3. Osnabrugge RL, Mylotte D, Head SJ, Van Mieghem NM, Nkomo V T, Le Reun CM, et al. Aortic stenosis in the elderly: Disease prevalence and number of candidates for transcatheter aortic valve replacement: A meta-analysis and modeling study. J Am Coll Cardiol 2013; 62:1002-12.

4. Cribier A, Eltchaninoff $\mathrm{H}$, Bash A, Borenstein N, Tron C, Bauer F, et al. Percutaneous transcatheter implantation of an aortic valve prosthesis for calcific aortic stenosis: First human case description. Circulation 2002; 106:3006-8.

5. Smith CR, Leon MB, Mack MJ, Miller DC, Moses JW, Svensson LG, et al. Transcatheter versus surgical aortic-valve replacement in high-risk patients. N Engl J Med 2011; 364: 2187-98. 
6. Kashiyama N, Kuratani T, Torikai K, Maeda K, Toda K, Miyagawa S, et al. Urgent transcatheter aortic valve replacement for severe aortic valve stenosis with acute decompensated heart failure: Report of a case. Surg Today 2015; 45:911-4.

7. Landes $U$, Orvin $K$, Codner $P$, Assali A, Vaknin-Assa $H$, Schwartznberg $S$, et al. Urgent transcatheter aortic valve implantation in patients with severe aortic stenosis and acute heart failure: Procedural and 30-day outcomes. Can J Cardiol 2016; 32:726-31.

8. Kapadia SR, Goel SS, Yuksel U, Agarwal S, Pettersson G, Svensson LG, et al. Lessons learned from balloon aortic valvuloplasty experience from the pre-transcatheter aortic valve implantation era. J Interv Cardiol 2010; 23:499-508.

9. Nishimura RA, Otto CM, Bonow RO, Carabello BA, Erwin JP, Guyton RA, et al. 2014 AHA/ACC guideline for the management of patients with valvular heart disease: A report of the American College of Cardiology / American Heart Association Task Force on Practice Guidelines. Circulation 2014; 129:2440-92.

10. Kolte D, Khera S, Vemulapalli S, Dai D, Heo S, Goldsweig AM, et al. Outcomes following urgent / emergent transcatheter aortic valve replacement: Insights from the STS/ACC TVT registry. JACC Cardiovasc Interv 2018; 11:1175-85.

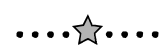

